\title{
Terahertz radiation enhancement in photoconductive antennas with embedded split-ring resonators
}

\author{
Hu Deng (邓 琥) $)^{1,2}$, Weiwei Qu (屈薇薇) ${ }^{1}$, Quancheng Liu (刘泉澄) ${ }^{1}$, Zhixiang Wu (武志翔) ${ }^{1}$, \\ and Liping Shang (尚丽平) $)^{1,2, *}$
}

${ }^{1}$ School of Information Engineering, Southwest University of Science and Technology, Mianyang 621010, China

${ }^{2}$ Joint Laboratory for Extreme Conditions Matter Properties, Southwest University of Science and Technology, Mianyang 621010, China

*Corresponding author: shangliping@swust.edu.cn

Received June 27, 2020; accepted August 18, 2020; posted online September 30, 2020

\begin{abstract}
This Letter proposes a novel method for enhancing terahertz $(\mathrm{THz})$ radiation from microstructure photoconductive antennas (MSPCA). We present two types of MSPCA, which contain split-ring resonators (SRRs) and dipole photoconductive antennas (D-PCAs). The experimental results reveal that when the femtosecond laser is pumping onto the split position of the SRR, the maximum $\mathrm{THz}$ radiation power is enhanced by 92 times compared to pumping at the electrode edge of the D-PCA. Two $\pi$ phase shifts occur as the pumping laser propagates from the negative electrode to the positive electrode. Analysis shows that photoinduced carrier charges move within the split position of the SRR.
\end{abstract}

Keywords: terahertz; microstructure photoconductive antenna; split-ring resonators.

doi: 10.3788/COL202018.113701.

In recent years, the most popular mechanisms for generating broadband terahertz $(\mathrm{THz})$ radiation are based upon optical rectification ${ }^{[\underline{1}]}$, surface generation ${ }^{[2]}$, and photoconductivity ${ }^{[3]}$. The latter approach is common, but it has a lower radiation power than the others ${ }^{[4]}$. Therefore, improving the radiation power of the photoconductive mechanism is an important capability that has significant practical ramifications-65. To achieve this enhancement in photoconductive antennas (PCA), researchers have proposed full-wave numerical techniques based on a coupled solution to the hydrodynamic transport equations, Poisson's drift diffusion, and Maxwell's equations ${ }^{[\mathbb{Z}]}$. These can simulate PCA with various active region geometries ${ }^{[8]}$. Researchers have also discussed the appropriate numerical values for impedance matching conditions and experiments to improve the efficiency of $\mathrm{PCA}^{[9-10]}$. The latter include a thin semiconductor layer sandwiched between a dielectric hyper hemispherical lens and a metal substrate $\stackrel{[11]}{=}$, a quasi-three-dimensional (3D) post-array $\stackrel{[12]}{- \text {, }}$ and a dielectric structure with periodic GaAs strips grown at low temperatures $\underline{[13]}$. A high efficiency $\mathrm{THz}$ pulse can be generated by pumping near-infrared light on large-area PCAs ${ }^{[14]}$, nano-plasmonic PCAs with metal nano-islands $\frac{[15]}{}$, or interdigitated PCAs based on plasmon electrodes $\stackrel{[16]}{-}$. A lot of semiconductor materials - such as single crystal and polycrystalline ZnSe substrates $\underline{[17]}$, III-V semiconductors and graphene $\stackrel{[18]}{-}$, epitaxial embedded rare-earth arsenide (ErAs and LuAs) nanoparticles in superlattice structures $\stackrel{[19]}{\text { — }}$ were researched to enhance $\mathrm{THz}$ radiation. PCAs using InGaAs-InAlAs multilayer heterostructures ${ }^{[20]}$ and SI-GaAs and n-GaAs wafer surfaces grown with aluminium nitride (AlN) thin films $\stackrel{[21]}{ }$ were proposed to achieve this enhancement. The structures of the $\mathrm{THz}$ PCA studied include optical nano-antennas ${ }^{[22]}$, fractal geometries ${ }^{[23]}$, tapered helix monopole $\stackrel{[24]}{-}$ array hexagonal metal nanostructures $\stackrel{[25]}{ }$, large area interdigitated ${ }^{[26]}$, 3D plasmon contact electro$\operatorname{des} \stackrel{[27]}{ }$, nano-structured electrodes ${ }^{[28]}$, thin-film plasmon electrodes $\stackrel{[22]}{ }$, and plasmon contact electrodes $\frac{[30]}{}$. In this Letter, we propose novel microstructure PCAs (MSPCA) that consist of split-ring resonators (SRRs) and dipole PCAs (D-PCAs). These MSPCAs provide a new way to robustly enhance the radiation power; we obtain $\pi$ phase shift and raise the maximum bias voltage by changing the pump position of the femtosecond laser.

The D-PCA with SI-GaAs as the photoconductive material is illustrated in Fig. 1(a). The dipole electrode width is $20 \mu \mathrm{m}$, and the inter-electrode gap is $80 \mu \mathrm{m}$. The structure of the SRR is shown in Fig. 1(b); this (a)

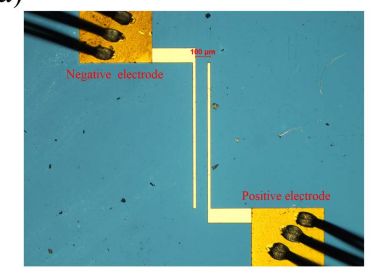

(c)

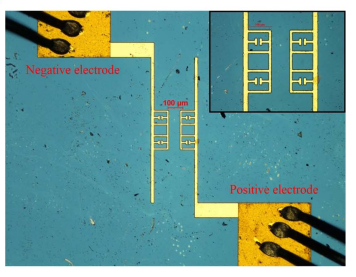

(b)

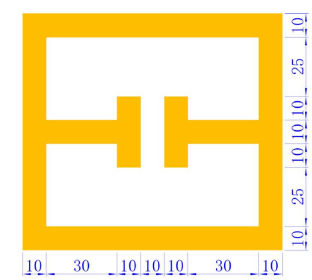

(d)

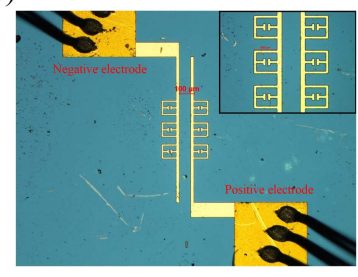

Fig. 1. D-PCA and MSPCA. (a) Micrograph of D-PCA. (b) Structure and parameters of SRRs. (c) Micrograph of MSPCA-A. (d) Micrograph of MSPCA-B. 


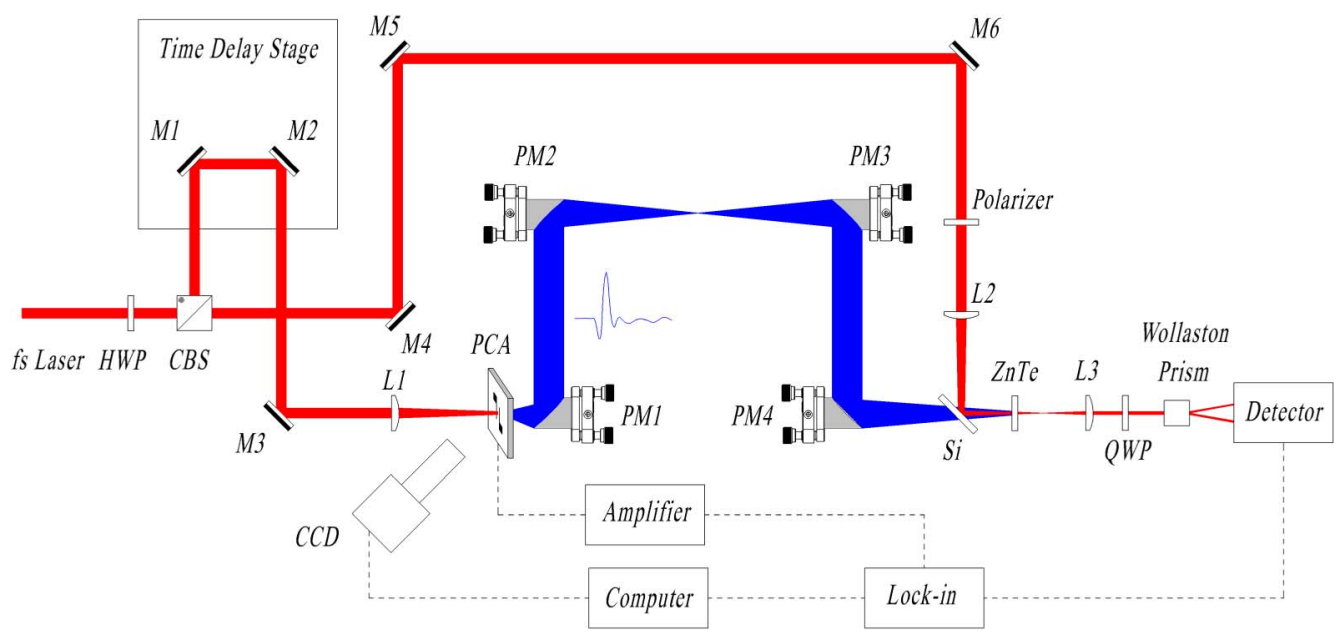

Fig. 2. Optical propagation trajectory of $\mathrm{THz}$ time domain spectroscopy system.

is widely used for optical modulation of $\mathrm{THz}$ waves. When a plane wave is incident perpendicularly to the surface of the SRR, the incident electric field excites the localized electromagnetic modes of the SRR. The SRR has a symmetrical structure with a length of $110 \mu \mathrm{m}$, a width of $100 \mu \mathrm{m}$, and a gap width of $10 \mu \mathrm{m}$. The SRRs with $80 \mu \mathrm{m}$ inter-electrode gap were coupled on the D-PCA in different arrangements to create different MSPCAs [Figs. 1(c) and 1(d)]. The D-PCA and MSPCAs were not coupled to a silicon lens.

The D-PCA, MSPCA-A, and MSPCA-B are tested with a $\mathrm{THz}$ time domain spectroscopy system, as illustrated in Fig. 2. A camera was added to monitor the spot size and pump position of the femtosecond laser on the PCA. The PCA has been placed on the $3 \mathrm{D}$ translation stage. We moved the $3 \mathrm{D}$ translation stage so that the femtosecond laser is pumped at different positions of the PCA. The key parameters of the pumping laser are listed as follows: wavelength is $800 \mathrm{~nm}$, pulse width is $92 \mathrm{fs}$, repetition frequency is $80 \mathrm{MHz}$, power is $60 \mathrm{~mW}$, and the diameter is $50 \mu \mathrm{m}$. The frequency of the lock-in amplifier and the time constant were $4.132 \mathrm{kHz}$ and $300 \mathrm{~ms}$, respectively, whereas the temperature and the humidity of the environment were $22^{\circ} \mathrm{C}$ and $40 \%$ relative humidity $(\mathrm{RH})$, respectively. The bias voltage of the PCA is the maximum output voltage without the amplifier (HVM500) being overloaded. This is because the amplifier's maximum output current at the bias voltage was $25 \mathrm{~mA}$.

The test steps of the PCA are listed as follows.

Step 1. We pumped the femtosecond laser at the interelectrode gap and electrode edge of the D-PCA, respectively, then adjusted the output voltage of the amplifier to make the bias voltage of the D-PCA the maximum value, and finally measured the characteristics of the $\mathrm{THz}$ radiation.

Step 2. The MSPCA-A and MSPCA-B substituted the D-PCA with invariant experimental conditions. We moved the pump laser from outside the negative electrode of the MSPCA to the positive electrode horizontally with a $10 \mu \mathrm{m}$ stepping interval at a different horizontal position successively, as shown in Figs. 3(a) and 3(b), then ensured that the bias voltage of MSPCA is the maximum value, and finally measured the characteristics of the $\mathrm{THz}$ radiation at different pumping positions.

With the femtosecond laser pumped onto the interelectrode gap of the MSPCA and the inter-electrode gap and electrode edge of the D-PCA, there are slight differences in the $\mathrm{THz}$ time domain spectroscopy, as shown as Fig. 4(a), between the amplitude and normalized amplitude of the PCA spectra. How the PCA $\mathrm{THz}$ wave radiates can be explained as follows. When the femtosecond laser is pumped at the inter-electrode, photoinduced carriers are generated from the semiconductor materials. These carriers move in the direction of the inter-electrode bias electric field and generate the photocurrent that radiates the $\mathrm{THz}$ waves. When the femtosecond laser pumps onto the inter-electrode gap of the MSPCA, the $\mathrm{THz}$ radiation includes two kinds of radiation: one directly radiated to the distant field by the accelerated motion of the photoinduced carriers in the bias electric field, and the other one that is scattered to the distant field by the energy coupled to the local electromagnetism mode. Due to the delay effects of the local electromagnetism mode to (a)

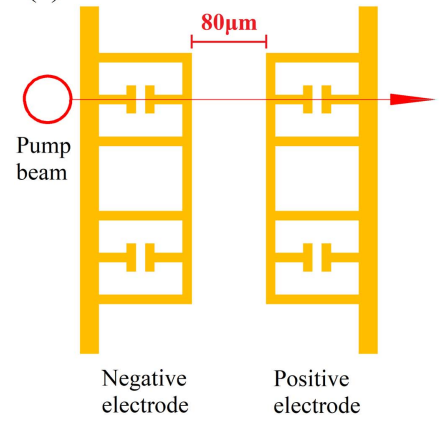

(b)

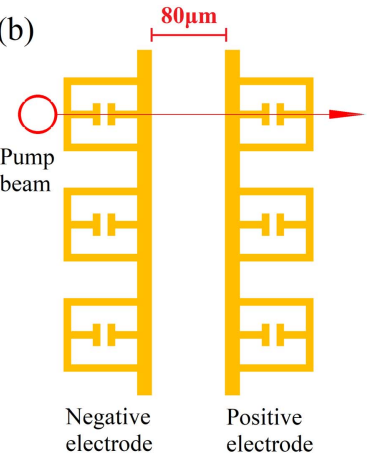

Fig. 3. Pumping position of femtosecond laser at (a) MSPCA-A and (b) MSPCA-B. 

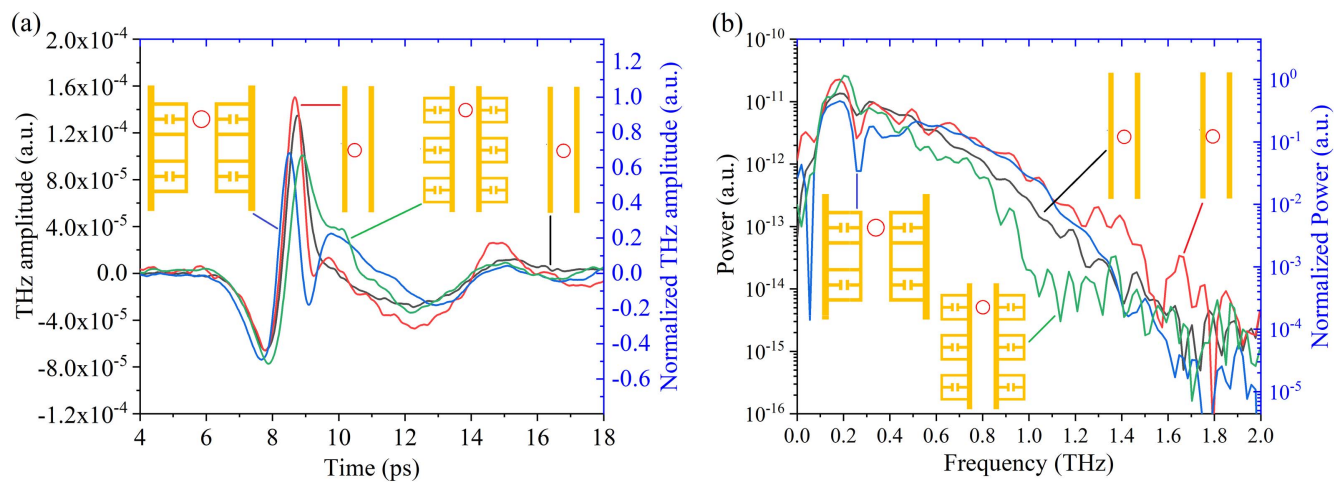

Fig. 4. THz characteristics of femtosecond laser pumping on the D-PCA, MSPCA-A, and MSPCA-B. Comparison of (a) amplitude and normalized amplitude of $\mathrm{THz}$ time domain spectra, and (b) power and normalized power of $\mathrm{THz}$ frequency domain spectra.

$\mathrm{THz}$ radiation pulse conversion, these electromagnetic pulses will interfere and superimpose in the distant field to produce the $\mathrm{THz}$ radiation. Consequently, the characteristics of local electromagnetism modes play an important role in the $\mathrm{THz}$ radiation emitted from the MSPCA. As illustrated in Fig. 4(b), the maximal radiation power is $1.36 \times 10^{-11}$ (a.u.) and $2.26 \times 10^{-11}$ (a.u.) with the femtosecond laser pumped on the inter-electrode gap and electrode edge of the D-PCA, respectively. The results show that more enhanced $\mathrm{THz}$ waves are generated when the femtosecond laser is pumped onto the electrode edge of the electrode. Therefore, in this Letter, we describe the $\mathrm{THz}$ radiation power (the normalized power is one) generated by the electrode edge of the femtosecond laser pumped D-PCA as a reference for comparison. Meanwhile, the maximal radiation power is $1.02 \times 10^{-11}$ (a.u.) and $2.61 \times 10^{-11}$ (a.u.) with the femtosecond laser pumped on the inter-electrode gap of MSPCA-A and MSPCA-B, respectively. The maximum of the normalized power is 0.45 and 1.15 with the femtosecond laser pumped on the inter-electrode gap of the MSPCA-A and the interelectrode gap of MSPCA-B, respectively. The radiation power is a relative value without any units, which is computed by fast Fourier transform (FFT) in the $\mathrm{THz}$ time domain data.

Compared to the femtosecond laser pumping on the electrode edge of the $\mathrm{D}-\mathrm{PCA}$, the $\mathrm{THz}$ radiation is noticeably enhanced. The absolute value and normalized value of $\mathrm{THz}$ amplitude and power are illustrated in Fig. $\underline{5}$. The plotted lines also oscillate when the femtosecond laser pumps onto the split positions of SRR on the MSPCAA and MSPCA-B, as shown in Figs. 5(a) and 5(c). This phenomenon demonstrates that the power within a magneto static resonance mode could be released gradually by a scattering loss. As illustrated in Figs. $\underline{5(\mathrm{~b})}$ and $\underline{5(\mathrm{~d})}$, the maximum radiation power was $2.08 \times 10^{-9}$ (a.u.) and $1.28 \times 10^{-9}$ (a.u.) when the pumping laser was incident on the split positions of the SRR on MSPCA-A and MSPCA-B, respectively. Experimental results demonstrate that the maximum power of the $\mathrm{THz}$ frequency domain spectrum can be enhanced by 92 and 56 times, respectively, by our proposed method. In addition, the low frequency power at $0.2-0.4 \mathrm{THz}$ is increased appreciably. This indicates that the low frequency peak corresponds to the fundamental mode of the SRR resonance. Here, the electric field is confined at the split position, while the magnetic field is restricted at the surrounding of the SRR; this demonstrates the basic characteristics of a magneto static resonance mode.

Compared to the case where the laser is pumped onto the left $5 \mu \mathrm{m}$ of the center of split positions of the SRR on MSPCA-A or MSPCA-B, the THz time domain spectrum has an opposite phase when the pumping laser is incident on the right $5 \mu \mathrm{m}$ of the center of split positions of the SRR on MSPCA-A [Fig. 6(a)] or MSPCA-B [Fig. 6(b)]. In addition, the waveforms of each exhibit certain symmetry. The patterns of $\mathrm{THz}$ time domain spectral peak-to-peak value are analyzed when the pumping laser is moved from the outside $20 \mu \mathrm{m}$ of the negative electrode to the positive electrode, as shown as Figs. 6(c) and 6(d). The experiments reveal that THz time domain phase shift of $\pi$ occurs twice at the split position of SRR, and the $\mathrm{THz}$ time domain spectral power results from pumping the laser onto the split position of the SRR; this is far greater than by pumping the laser onto any other position of the MSPCA.

Figure $\underline{7(\mathrm{a})}$ shows the cases where the femtosecond laser is pumped onto the positive electrode, the negative electrode, and the inter-electrode gap of MSPCA$\mathrm{B}$, where the maximum (or minimum) intensities of the $\mathrm{THz}$ time domain spectrum were $9.32 \times 10^{-4}$ (a.u.), $-6.74 \times 10^{-4}$ (a.u.), and $1.03 \times 10^{-4}$ (a.u.), and the bias voltages were $74 \mathrm{~V}, 57 \mathrm{~V}$, and $42 \mathrm{~V}$, respectively. Figure $\underline{7(b)}$ shows the cases where the femtosecond laser is pumped onto the positive electrode, the negative electrode, and the inter-electrode gap of MSPCA-B, where the maximum (or minimum) intensities of the $\mathrm{THz}$ time domain spectrum were $8.54 \times 10^{-4}$ (a.u.), $-5.60 \times 10^{-4}$ (a.u.), and $1.01 \times 10^{-4}$ (a.u.), and the bias voltages were $68 \mathrm{~V}, 57 \mathrm{~V}$, and $53 \mathrm{~V}$, respectively.

By comparing Fig. $\underline{7(\mathrm{a})}$ with Fig. $\underline{6(\mathrm{c})}$ and Fig. $\underline{7(\mathrm{~b})}$ with Fig. $6(\mathrm{~d})$, it can be supposed that the peak-to-peak value of the time domain spectrum is not proportional to the 

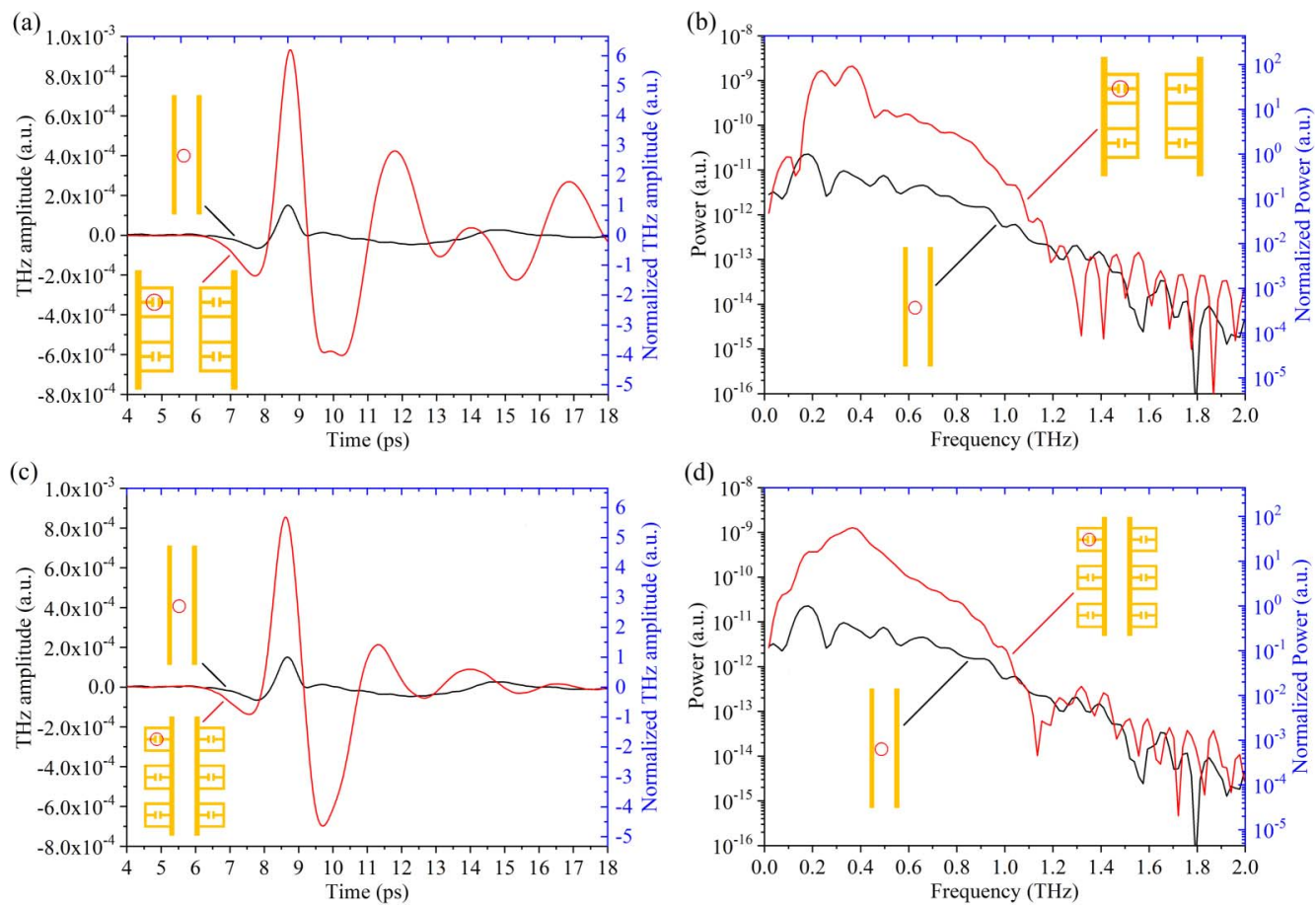

Fig. 5. Comparison of the characteristics of the THz radiation for femtosecond laser pumping on the split positions of SRR of MSPCA and on the electrode edge of the D-PCA. (a) THz amplitude and normalized THz amplitude of MSPCA-A and D-PCA, (b) THz power and normalized THz power of MSPCA-A and D-PCA, (c) THz amplitude and normalized THz amplitude of MSPCA-B and D-PCA, and (d) THz power and normalized THz power of MSPCA-B and D-PCA.

bias voltage. These figures reveal that the bias voltages on the positive and negative electrodes are basically constant, while the peak-to-peak value of the time domain spectrum varies significantly. The peak-to-peak value of the time domain spectrum primarily depends on both the pumping position of the laser and the local electromagnetic mode.
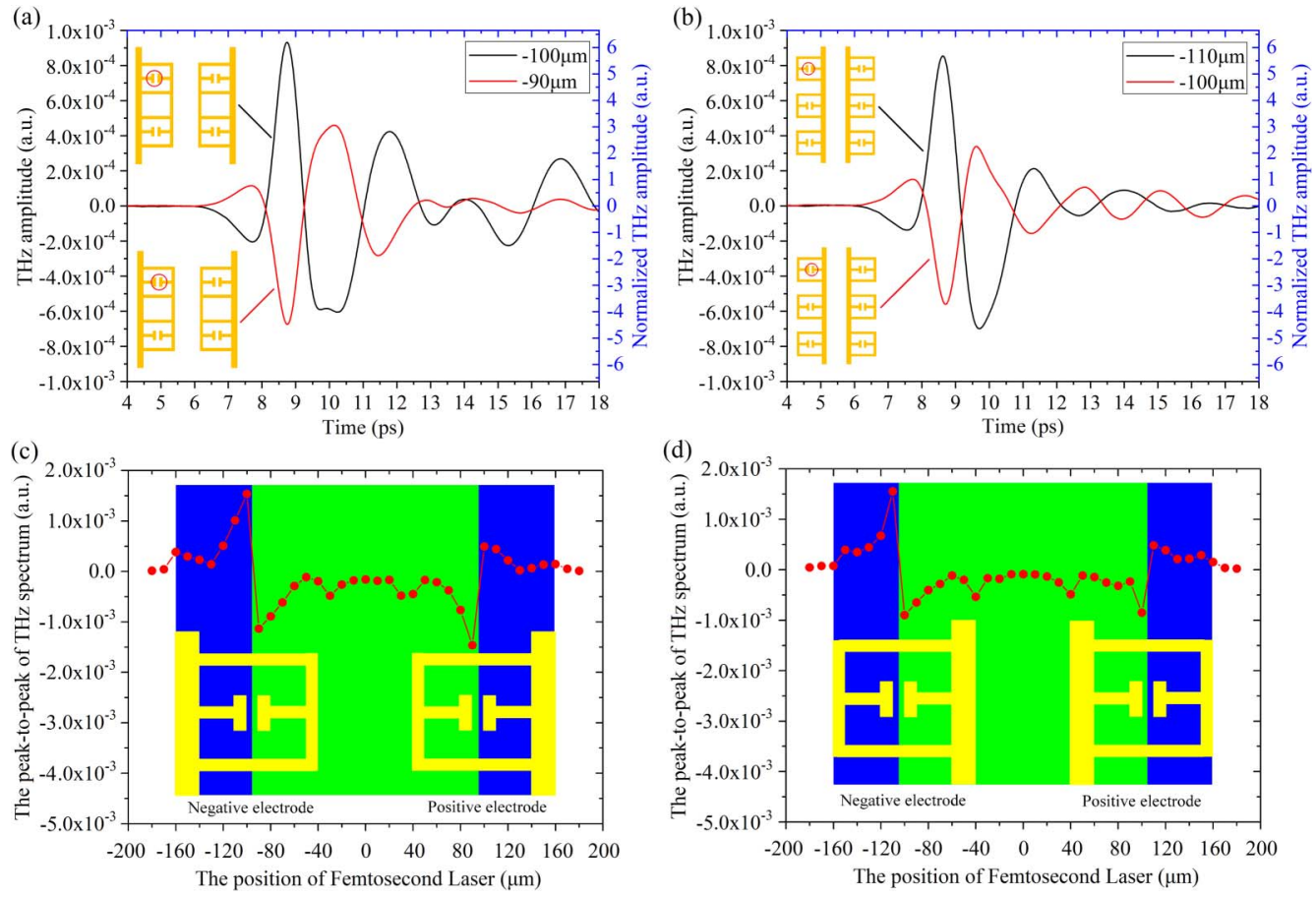

Fig. 6. Comparison of $\mathrm{THz}$ time domain spectrum for femtosecond laser pumping at different positions. THz amplitude and normalized THz amplitude of (a) MSPCA-A when the pumping light is incident on $-90 \mu \mathrm{m}$ and $-100 \mu \mathrm{m}$ and (b) MSPCA-B when the pumping light is incident on $-110 \mu \mathrm{m}$ and $-100 \mu \mathrm{m}$. Pattern of $\mathrm{THz}$ time domain spectral peak-to-peak value of (c) MSPCA-A and (d) MSPCA-B. 
(a)

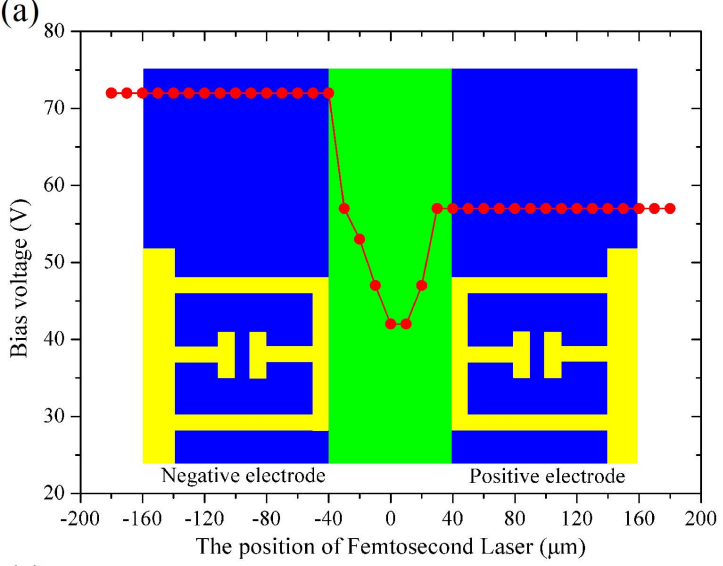

(c)

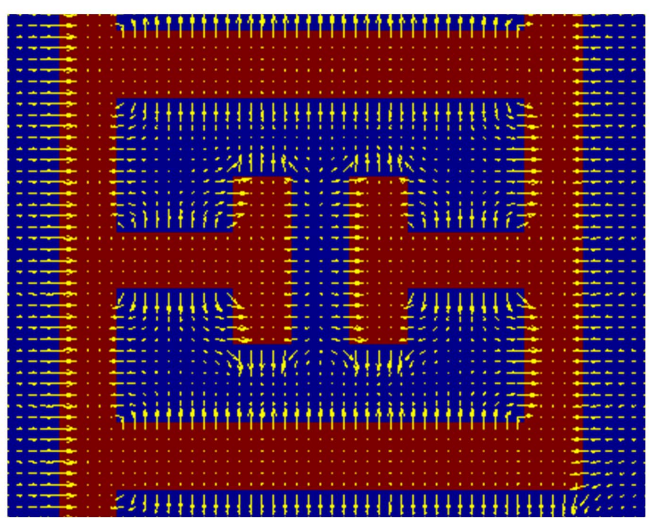

(b)

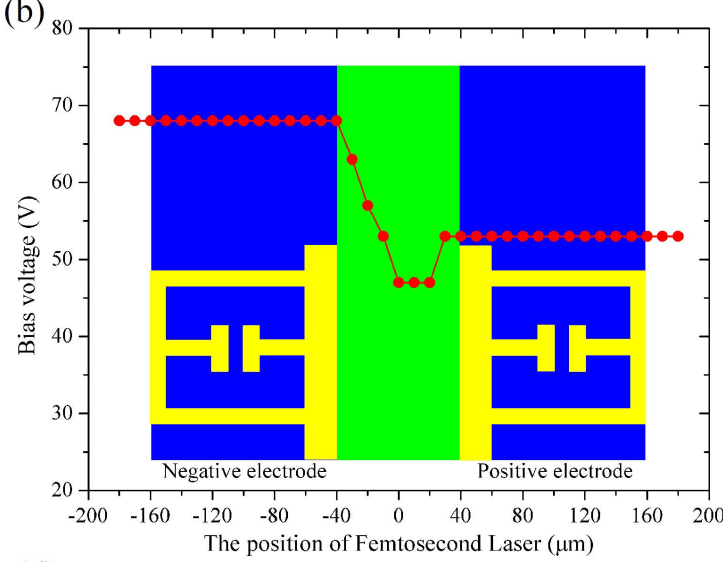

(d)

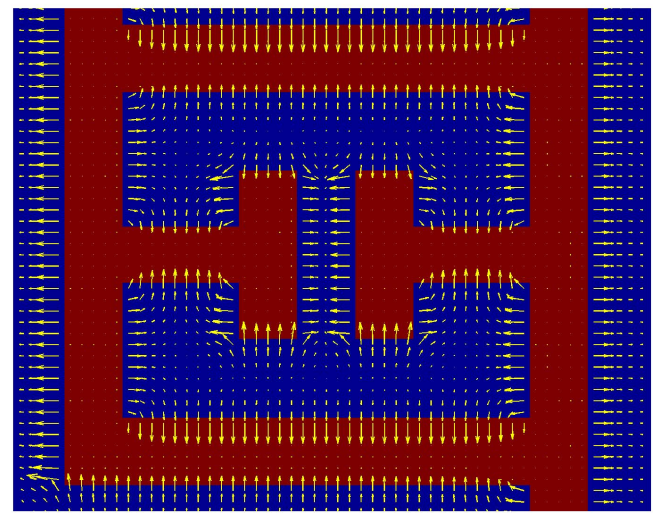

Fig. 7. Patterns at the maximum bias voltage when the femtosecond laser is pumped onto different positions of (a) MSPCA-A and (b) MSPCA-B. The electrostatic field of the (c) positive electrode and (d) negative electrode of the MSPCA.

In addition, the bias voltage on the positive electrode, the negative electrode, and the inter-electrode gap could have an impact on the peak-to-peak value of the time domain spectrum.

The experimental results reveal that the maximum bias voltage rises when the pumping laser is on the positive or negative electrode, while the maximum bias voltage remains at lower levels for laser pumping at the interelectrode gap. Moreover, when the pumping laser is on the negative electrode, the bias voltage is higher than when the pumping laser is on the positive electrode. The $\mathrm{THz}$ electric intensity increases in the MSPCA, since the bias voltage increase is much greater than in D-PCA; this is proportional to the bias voltage $\stackrel{[31]}{-}$. Meanwhile, the electrostatic field between the positive and negative electrodes of the MSPCA is complex with a two-dimensional nonuniform electric field, as shown in Figs. $\underline{7(\mathrm{c})}$ and $\underline{7(\mathrm{~d})}$. Furthermore, the two sides of the split of SRR are on the same electrode; hence, the direction of the electrostatic field in the left and right parts is reversed. The pumping laser shifts $\pi$ phase when moving from left to right of this split position.

In conclusion, our method is novel and significant because of the production of $\mathrm{THz}$ radiation that differs in character to traditional D-PCAs. The analysis reveals that only the direction of motion of the photoinduced carrier charges can lead to the $\pi$ phase shift of $\mathrm{THz}$ radiation waveform. The electrostatic field of the traditional D-PCA is approximately a one-dimensional uniform electric field with one direct motion of photoinduced carriers, which will not change dramatically with the position of a pumping laser. Unlike D-PCA, the electrostatic field between the positive and negative electrode of the MSPCA is a two-dimensional non-uniform electric field. Accordingly, the photoinduced carriers move within the split position of the SRR rather than the interelectric gap of the MSPCA. Importantly, our MSPCA outputs enhanced $\mathrm{THz}$ radiation power. We have analyzed the reasons for its $\mathrm{THz}$ enhancement. First, the SRR has localized the THz energy in the low frequency band, which has caused a sharp increase in the low frequency radiation power. Second, the photogenerated carriers have moved within the split position of the SRR. Third, the bias voltage of the MSPCA has been increased, making the $\mathrm{THz}$ radiation power significantly improved. The enhanced $\mathrm{THz}$ radiation power of the proposed method could be a solution for space charge shielding and the breakdown between electrodes. This method also has extensive potential for applications relating to biomedicine, material analysis, and other $\mathrm{THz}$ uses.

This work was supported by the National Natural Science Foundation of China (No. 11872058), the National Defense Basic Scientific Research Program of 
China (Nos. JCKY2018404C007, JSZL2017404A001, and JSZL2018204C002), and the Sichuan Science and Technology Program of China (No. 2019YFG0114).

\section{References}

1. S. L. Chuang, S. Schmitt-Rink, B. I. Greene, P. N. Saeta, and A. F. Levi, Phys. Rev. Lett. 68, 102 (1992).

2. X. C. Zhang, B. B. Hu, J. T. Darrow, and D. H. Auston, Appl. Phys. Lett. 56, 1011 (1990).

3. G. Mourou, C. V. Stancampiano, A. Antonetti, and A. Orszag, Appl. Phys. Lett. 39, 295 (1981).

4. J. T. Darrow, B. B. Hu, X. C. Zhang, and D. H. Auston, Opt. Lett. 15, 323 (1990).

5. H. Zhang, Z. Zhang, X. Zhao, X. Zhang, T. Zhang, C. Cao, and Y. Yu, Chin. Opt. Lett. 16, 103001 (2018).

6. C. Ruan, D. Kong, J. Dai, K. Chen, S. Guo, and X. Wu, Chin. Opt. Lett. 17, 073001 (2019).

7. M. A. Khorrami, S. El-Ghazaly, H. Naseem, and S. Q. Yu, IEEE Trans. Terahertz Sci. Technol. 4, 101 (2014).

8. R. Emadi, N. Barani, R. Safian, and A. Z. Nezhad, J. Infrared Millim. Terahertz Waves 37, 1069 (2016).

9. S. Preu, J. Infrared Millim. Terahertz Waves 35, 998 (2014).

10. D. V. Lavrukhin, A. E. Yachmenev, A. Y. Pavlov, R. A. Khabibullin, Y. G. Goncharov, I. E. Spektor, G. A. Komandin, S. O. Yurchenko, N. V. Chernomyrdin, K. I. Zaytsev, and D. S. Ponomarev, Semicond. Sci. Tech. 34, 034005 (2019).

11. M. I. Bakunov, R. V. Mikhaylovskiy, and M. Tani, Opt. Express 18, 22406 (2010).

12. N. Koja, J. C. Young, and T. Suzuki, Appl. Phys. A 120, 479 (2015).

13. M. Khorshidi and G. Dadashzadeh, J. Infrared Millim. Terahertz Waves 38, 609 (2017).

14. M. Beck, H. Schaefer, G. Klatt, J. Demsar, S. Winnerl, M. Helm, and T. Dekorsy, Opt. Express 18, 9251 (2010).

15. S. G. Park, Y. Choi, Y. J. Oh, and K. H. Jeong, Opt. Express 20, 25530 (2012).
16. A. Singh and S. S. Prabhu, Opt. Express 23, 1529 (2015).

17. X. Ropagnol, R. Morandotti, T. Ozaki, and M. Reid, IEEE Photon. J. 3, 174 (2011).

18. T. Otsuji, T. Watanabe, S. A. B. Tombet, A. Satou, W. M. Knap, V. V. Popov, M. Ryzhii, and V. Ryzhii, IEEE Trans. Terahertz Sci. Technol. 3, 63 (2013).

19. N. T. Yardimci, R. Salas, E. M. Krivoy, H. P. Nair, S. R. Bank, and M. Jarrahi, Opt. Express 23, 32035 (2015).

20. A. Bockelt and B. Vidal, IEEE Trans. Terahertz Sci. Technol. 7, 107 (2017).

21. R. B. Jaculbia, M. H. M. Balgos, N. S. Mangila, M. A. C. Tumanguil, E. S. Estacio, A. A. Salvador, and A. S. Somintac, Appl. Surf. Sci. 303, 241 (2014).

22. S. Lepeshov, A. Gorodetsky, A. Krasnok, E. Rafailov, and P. Belov, Laser Photon. Rev. 11, 1600199 (2017).

23. P. Maraghechi and A. Y. Elezzabi, IEEE J. Sel. Top. Quantum Electron. 19, 8400310 (2013).

24. V. Varlamava, F. Palma, P. Nenzi, and M. Balucani, IEEE Trans. Terahertz Sci. Technol. 4, 360 (2014).

25. A. Jooshesh, L. Smith, M. Masnadi-Shirazi, V. Bahrami-Yekta, T. Tiedje, T. E. Darcie, and R. Gordon, Opt. Express 22, 27992 (2014).

26. K. Maussang, A. Brewer, J. Palomo, J. M. Manceau, R. Colombelli, I. Sagnes, J. Mangeney, J. Tignon, and S. S. Dhillon, IEEE Trans. Terahertz Sci. Technol. 6, 20 (2016).

27. S. H. Yang, M. R. Hashemi, C. W. Berry, and M. Jarrahi, IEEE Trans. Terahertz Sci. Technol. 4, 575 (2014).

28. K. Moon, I.-M. Lee, J.-H. Shin, E. S. Lee, N. Kim, W.-H. Lee, H. Ko, S.-P. Han, and K. H. Park, Sci. Rep. 5, 13817 (2015).

29. N. Burford and M. El-Shenawee, J. Opt. Soc. Am. B 33, 748 (2016).

30. N. T. Yardimci, S. H. Yang, C. W. Berry, and M. Jarrahi, IEEE Trans. Terahertz Sci. Technol. 5, 223 (2015).

31. J. T. Darrow, X.-C. Zhang, D. H. Auston, and J. D. Morse, IEEE J. Quantum Elect. 28, 1607 (1992). 